RUB-TPII-21/95

\title{
Elusive exotic states
}

\author{
Hyun-Chul Kim * \\ Institut für Theoretische Physik II, \\ Postfach 102148, Ruhr-Universität Bochum, \\ D-44780 Bochum, Germany \\ Mikhail Shmatikov ${ }^{\dagger}$ \\ Russian Research Center "Kurchatov Institute", 123182 Moscow, Russia
}

(July 1995)

\begin{abstract}
The existence of flavor exotic $Q Q \bar{q} \bar{q}$ molecular-type states is investigated. An attractive force between two pseudoscalar $H=(Q \bar{q})$ heavy meson is generated by (correlated) two-pion exchange. The emergence of a (loosely) bound state depends crucially on the value of the coupling constant $g$ of the $H^{*} H \pi$ vertex. For a $g$ value calculated from the experimental upper limit on the width of the $D^{*}$ meson the considered mechanism alone is strong enough to generate a bound state in the $B B$ system while the $D D$ system is very close to become bound. Such states, if exist, are stable with respect to strong interactions. They may be observed as stable scalar particles with the mass $M \approx 2 m_{H}$ and flavor quantum number \pm 2 .
\end{abstract}

\footnotetext{
*e-mail address: kim@hadron.tp2.ruhr-uni-bochum.de

$\dagger$ †-mail address: msh@ofpnp.kiae.su
} 
At present basically all the hadrons, both baryons and mesons, can be safely classified as $q q q$ and $\bar{q} q$ quark states respectively. No reliable evidence for multiquark states but nuclei, which can be considered as weakly bound states of the baryons, has been found. One of the most clear-cut signals for such multiquark states would be an observation of hadronic states with exotic flavor quantum numbers. A promising hunting ground for such an exotics is the domain of heavy flavors, or more precisely, hadrons with the $Q Q \bar{q} \bar{q}$ structure where $Q$ is a heavy quark. The sought after hadronic states are known to exist in the $m_{Q} \rightarrow \infty$ limit [1] [3]. Quantitatively the heavy-mass limit can be recast in the form of an inequality

$$
\alpha_{s}^{2}\left(m_{Q}\right) m_{Q} \gtrsim \Lambda_{Q C D} .
$$

However, this inequality is satisfied for the $t$-quark only which, on the other hand, decays before the hadronization. For $Q=c, b$ quarks $Q Q \bar{q} \bar{q}$ hadrons may exist as weakly-bound systems of two $Q \bar{q}$ mesons.

A couple of $Q \bar{q}$ mesons may be bound, for the large enough mass of a heavy quark, by a comparatively weak force generated by one-pion exchange. The attractive feature of such a long-range potential is that it is calculable in a chiral perturbation theory. A molecule-type $H-H(H=Q \bar{q})$ hadron exists in the limit of the infinitely heavy mass $m_{Q}$ [3]. Corrections $\sim 1 / m_{Q}$, in the realistic case of $b$ - or $c$ - quarks, prove to be of importance. The investigation of such systems in [3 5 yielded rather controversial results. According to [3] the $D$-mesons are too light to be bound by the one-pion-exchange force, whereas a loosely bound state was found in the system of two $B$-mesons consisting of $B B^{*}$ and $B^{*} B^{*}$ with equal weights. At the same time the one-pion-exchange force between two heavy mesons was found in [4,5] to be too weak (or even repulsive) to produce a bound state. In contrast, systems with non-exotic quantum numbers were shown to have a rich spectrum of bound states: $D \bar{D}$ system is expected to have a bound state and the $B \bar{B}$ one possesses several such states with various spin-parity quantum numbers. It should be stressed that conclusions as to the existence of a bound state(s) rely heavily on the specific value of the strength constant $g$ of the $\pi$-meson coupling to a heavy meson which sets the overall scale of the interaction 
strength (see below).

In the limit $m_{Q} \rightarrow \infty$ mesons containing a single heavy quark come in degenerate doublets of pseudoscalar $(H)$ and vector $\left(H^{*}\right)$ mesons. Mass corrections being taken into account, the degeneracy in the total momentum is lifted off and the $H$ and $H^{*}$ mesons are to be treated separately. For this reason one-pion exchange is operative in the $H^{*} H^{*}$ and $H H^{*}$ (or $\bar{H}^{*} H^{*}$ and $\bar{H} H^{*}$ ) systems only and all the conclusions of [3] refer just to such systems.

In the present paper we investigate coupling and possible bound states of two pseudoscalar heavy mesons $H H$. There are two reasons arousing interest to such a system. First, since a pseudoscalar $H$-meson is lighter than its vector counterpart $H^{*}$, a bound $H-H$ state will be stable with respect to a strong decay. An $H^{*}-H^{*}$ system, spin-parity allowing, may decay into the $H H$ pair. Open decay channel brings additional repulsion which may destroy a bound state [6]. Another reason for investigating a system of two pseudoscalar meson has a dynamical nature. The vertex of pion coupling to a pair of the $H$-mesons apparently vanishing, the next-in-range force operative in this system is (correlated) two-pion exchange (CTPX). This force is known to provide the bulk of the attraction in the $N N$ system [7]. Thus it is of interest to investigate its effects in heavy-meson systems, and the $H H$ pair provides a testing ground where the CTPX forces are not obscured by the one-pion exchange.

The construction of the CTPX potential is described in the plethora of works (see 79 just to mention a few). The generic form of two-pion exchange is depicted in fig.1. A pair of pions in the $t$-channel may have various orbital momenta and isospin. We focus our attention on the $J^{\pi}(T)=0^{+}(0)$ channel, which is the most relevant for the system under consideration, since it is responsible for the strong sttraction. We will postpone the discussion of $J \geq 1$ contributions to the conclusion. As is well known from the works on the $N N$ interaction, the static potential for the scalar-isoscalar channel can be written by

$$
V_{2 \pi}(r)=-\frac{1}{\pi} \int_{4 m_{\pi}^{2}}^{t_{\max }} d t \rho(t) \frac{e^{-\sqrt{t r}}}{4 \pi r} .
$$




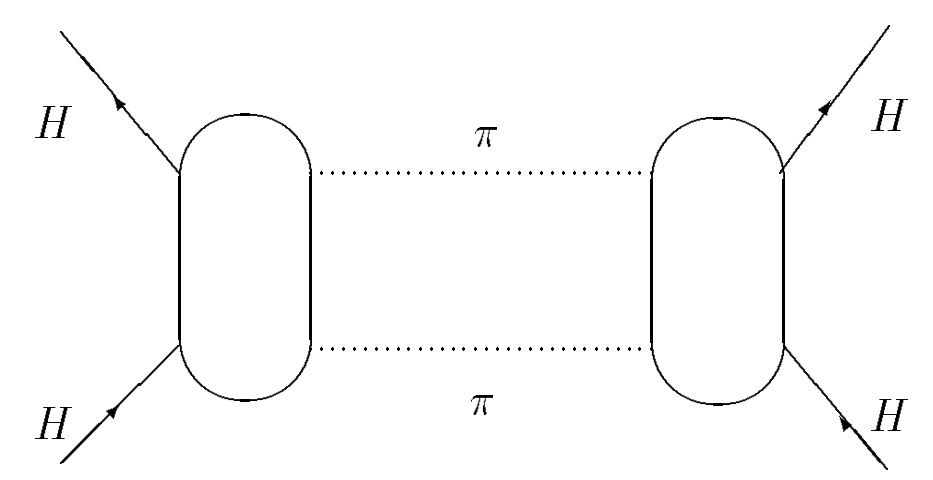

Fig.1: Two-pion intermediate state in the $H H \rightarrow H H$ scattering.

The dynamics of the intercation is controlled by the behavior of the spectral function $\rho(t)$. It vanishes at the two-pion treshold in the $t$-channel, i.e. at $t=4 m_{\pi}^{2}$. The upper integration limit $t_{\max }$ is usually chosen $t_{\max } \simeq 50 \mathrm{~m}_{\pi}^{2}$. Thus the CTPX potential is a superposition of Yukawa forces corresponding to the exchange by a meson with the $\sqrt{t}$ mass and the weight of this configuration is given by the corresponding value of the $\rho(t)$ spectral function.

The spectral function is in turn expressed, by means of the unitarity condition, in terms of the amplitude $\mathcal{A}$ of the $\bar{H} H \rightarrow \pi \pi$ process:

$$
\rho(t)=\frac{1}{32 \pi} \sqrt{\frac{t-4 m_{\pi}^{2}}{t}}|\mathcal{A}(t)|^{2} .
$$

Note that in contrast to the entirely familiar $\bar{N} N \rightarrow \pi \pi$ case there is no iterated Born term to be subtracted. In case of the $\bar{N} N \rightarrow \pi \pi$ process, the quasiempirical data in the pseudophysical region $\left(4 m_{\pi}^{2}<t<50 m_{\pi}^{2}\right)$ can be obtained by making an analytic continuation of the $\pi N$ scattering data. However, for lack of the quasiempirical information on the $\pi$-meson scattering off a heavy meson we are forced to apply a dynamical model.

Such a model developed in [10] for the $N N$ interaction was shown to reproduce with good accuracy available experimental data on scattering phase shifts in a wide energy region. The dynamical model tailored for the case of $\bar{H} H \rightarrow \pi \pi$ amplitude is shown in fig.2. 

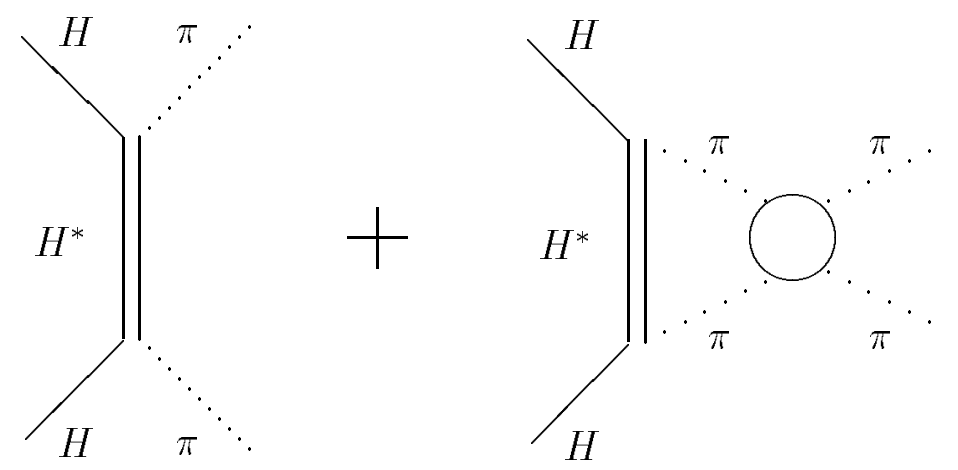

Fig.2: Dynamical model of the $\bar{H} H \rightarrow \pi \pi$ annihilation amplitude. Empty circle denotes the T-matrix of the $\pi \pi \rightarrow \pi \pi$ rescattering.

It consists of two components: the Born term where outgoing pions do not interact with each other and the one involving pion rescattering. The amplitude of the (half-off-shell) $\pi \pi \rightarrow \pi \pi$ interaction can be evaluated by using the meson-exchange model of $\pi \pi, \pi K$ and $K K$ processes [11]. As usual, a nonvanishing range of the strong interaction is taken into consideration by introducing a formfactor $F$ in the $H H^{*} \pi$ vertex. We choose the exponential form [12] for it:

$$
F\left(k^{2}\right)=\exp \left\{-\frac{k^{2}+M^{2}}{2 \Lambda}\right\}
$$

where $M$ is the mass of the exchange particle and $\Lambda$ denotes the cut-off parameter.

The overall strength scale of the (2) potential is established by the constant $g$ of the $\pi H^{*} H$ coupling. It is defined as follows 13

$$
\mathcal{L}=\frac{i}{2} g \operatorname{Tr}\left[H \gamma_{\mu} \gamma_{5}\left(\xi^{\dagger} \partial_{\mu} \xi-\xi \partial_{\mu} \xi^{\dagger}\right) \bar{H}\right]
$$

In principle, the value of $g$ could be determined from the width of the $H^{*} \rightarrow H \pi$ decay channel. However, because of small mass difference $\Delta m_{B}$ between $B$ and $B^{*}$ mesons $\left(\Delta m_{B} \approx 46\right.$ $\mathrm{MeV}$ ) this channel is closed for the $B^{*}$-meson. As to the system of (vector and pseudoscalar) $D$-mesons, at present the upper limit for the total width of the $D^{*}$-meson and partial width of the $D^{*} \rightarrow D \pi$ decay are measured [14]. Theoretical estimates of the $g$ value are controversial. The upper limit in both the chiral and the heavy quark limits in the leading $1 / N_{c}$ order reads 15]: 


$$
g \leq \frac{1}{\sqrt{2}}\left[1+\mathcal{O}\left(1 / m_{H}\right)+\mathcal{O}\left(1 / N_{c}\right)+\mathcal{O}\left(m_{\pi} m_{H}\right)\right]
$$

The numerical values utilized in [4,5] and [3], which ensured the existence of a (loosely) bound state, are equal to $g \approx 0.6$ and 0.7 respectively. These values were obtained by assuming that the upper experimental limit on the width of the $D^{*}$ meson is just the realistic value of the latter. Direct calculations of $g$ based on the QCD sum rules [16] yielded much smaller value: $g \approx 0.2$. Note that the $H^{*} H \pi$ vertex enters in the spectral function $\rho(t)$ four times, which implies that the CTPX potential $V_{2 \pi}$ (2) contains the $g^{4}$ factor. Thus the specific value of $g$ proves to be crucial for the (non)existence of a bound state of two pseudoscalar $H-H$ mesons.

Under these circumstances we have investigated the existence of the $H-H$ bound state and, if present, calculated the binding energy $E_{b}$ in a wide range of $g$ 's: $0 \leq g \leq 1$. In the numerical calculations of the CTPX potential (2) the value of $t_{\max }$ was chosen, as it is customary in the $N N$ system, equal to $t_{\max }=50 \mathrm{~m}_{\pi}^{2}$. The spectral function (3) being positive-defined, a decrease of the $t_{\max }$ may be compensated by the corresponding increase of the $g$ coupling constant. The cut-off parameter $\Lambda$, entering into the formfactor (田), was varied in the $\Lambda=1.5 \div 2.1 \mathrm{GeV}$ interval.

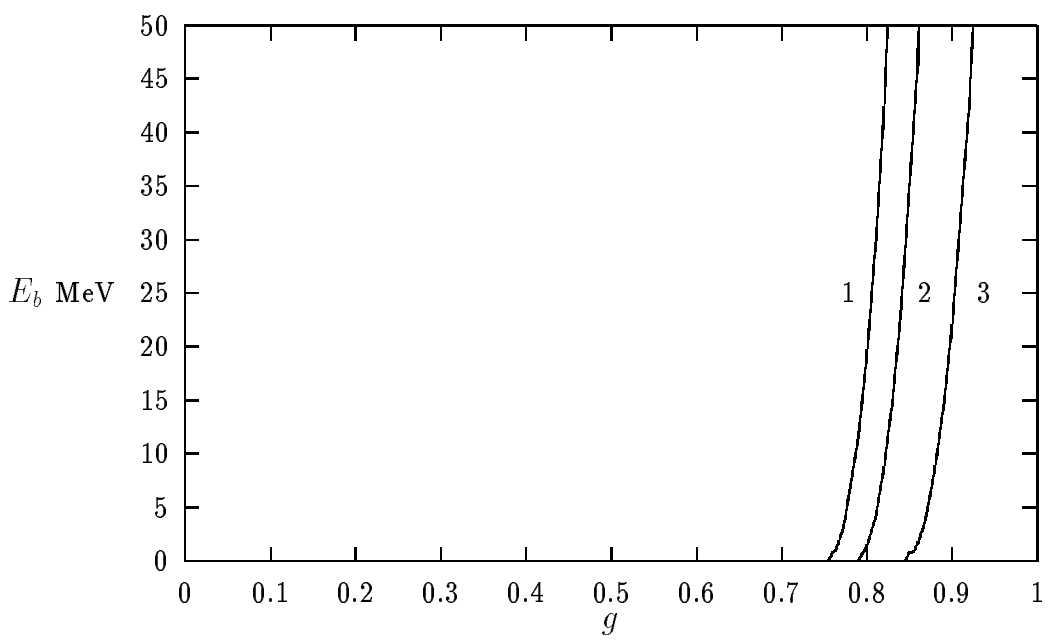

Fig.3: Dependence of the binding energy $E_{b}$ of two pseudoscalar $D$-mesons on the $D^{*} D \pi$ coupling constant $g$. Curves labeled 1,2 and 3 correspond to the cut-off paremeter $\Lambda=2.1$, 1.8 and $1.5 \mathrm{GeV}$ respectively. 
Results of calculations for the $D D$ system ( $S$-wave) are presented in fig.3. Note the (anticipated) sharp dependence of the binding energy on the $g$ value and its rather weak spread with the variation of $\Lambda$. A conclusion, which can be drawn from the investigation of the $g$ dependence, is that the bound state emerges at the value of $g \approx 0.75 \div 0.85$, i.e. at somewhat larger $g$ 's than the molecular states of refs. [4, [5, 3] ]. In the case of more heavy $B$-mesons situation proves to be more favorable (see fig.4). Here the bound state emerges, depending upon the cut-off parameter, in the $g \approx 0.58 \div 0.78$ range.

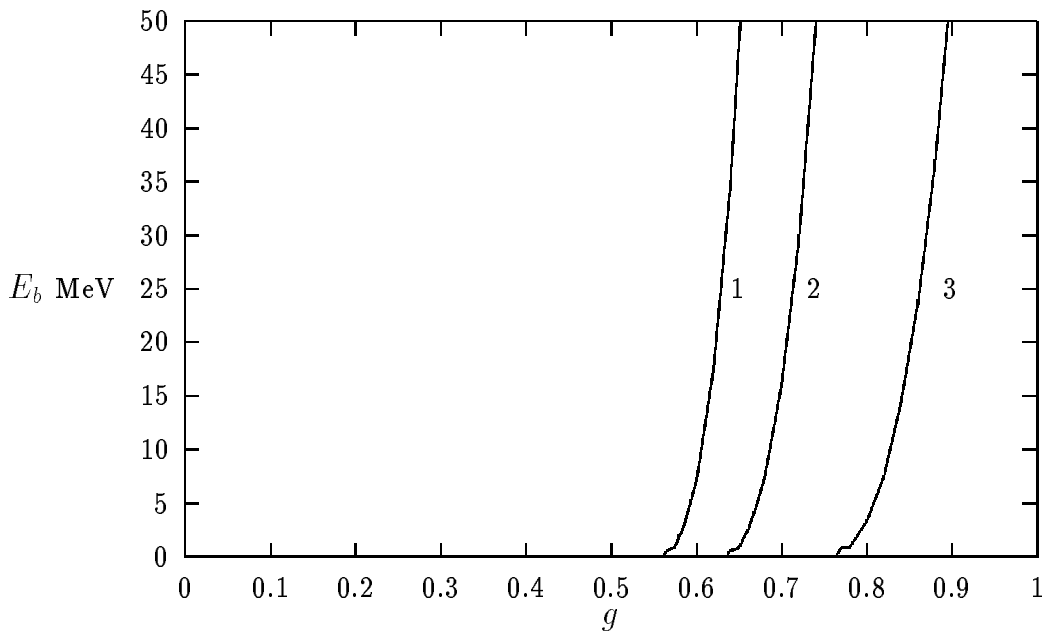

Fig.4: Dependence of the binding energy $E_{b}$ of two pseudoscalar $B$-mesons on the $B^{*} B \pi$ coupling constant $g$. Curves labeled 1,2 and 3 correspond to the cut-off paremeter $\Lambda=2.1$, 1.8 and $1.5 \mathrm{GeV}$ respectively

Hence, one can conclude that flavor exotic $D D$ (molecular) states are rather non-existing. Two pseudoscalar $B$ mesons become bound at about the same value of the $g$ coupling constant when the bound state in the $\bar{B}^{*} B^{*}$ and $\bar{B}^{*} B$ 近, 5 or $B^{*} B^{*}$ [3] systems emerges. For small $g \approx 0.2$ [16 molecular-type states with any meson content do not exist. It should be noted, that in contrast to the case of one-pion exchange which has the opposite (because of G-parity) sign in the $H^{*} H^{*}$ and $\bar{H}^{*} H^{*}$ systems, CTPX remains attractive in both mesonmeson and meson-antimeson systems. It implies that obtained results hold true also for the $\bar{D} D$ and $\bar{B} B$ (non-exotic) systems. Stated differently, provided the latter molecular states are observed, existence of their flavor exotic counterparts $(B B$ and $D D)$ is highly 
plausible. Considered mechanism is operative as well in similar system containing vector heavy mesons. The main difference is that one-pion exchange is operative in such systems. Then the attraction provided by the CTPX can either in combination with the one-pionexchange induced attraction or overwhelming repulsion, generate otherwise unobtainable molecular-type states.

We have considered the possible formation of molecular-type bound states of two pseudoscalar heavy mesons generated by the CTPX with the $t$-channel quantum numbers $J^{\pi}=0^{+}$. The effective potential $V_{2 \pi}$ is contributed as well by the CTPX with other quantum numbers $J^{\pi}=1^{-}$and $2^{+}$. However, the $\pi \pi$ interaction with such quantum numbers involves additional, as compared to the considered case, momenta of pions. The integration over the pionic loop (fig.2) will result then in a spectral function peaked at larger values of $t$ or, in the coordinate space, to the CTPX potential with smaller interaction range. Barring accidental emergence of a state with almost vanishing binding energy such states are expected to be compact ones. Their dynamics will be governed by the combined action of CTPX and QCD quark-quark interaction. Existence of bound states in the situation when both meson exchanges and quark coupling come into play will be considered elsewhere.

Summarizing we have investigated a possible existence of molecular-type bound states in flavor exotic $H H$ systems. In a sense it is an extension of the results obtained in [3] which predicted, in the $m_{Q} \rightarrow \infty$ limit, the existence of a bound heavy-meson state. However, $1 / m_{Q}$ corrections are of importance, necessitating the different approach to the vector $H^{*}$ and pseudoscalar $H$ heavy mesons. All the heavy-meson systems differ drastically from the one $(H H)$ considered in the present paper. First, their dynamics is controlled by the combined action of the one- and (correlated) two-pion exchange. It implies that besides the $H^{*} H \pi$ coupling constant one more strength coupling constant (in the $H^{*} H^{*} \pi$ vertex) plays an important role. Second, many of the states involving the vector $H^{*}$ heavy meson may decay due to the $H^{*} \rightarrow H$ transformation. The presence of an open decay channel may affect strongly the properties and the very existence of the sought after bound state [6]. At the same time coupling of the considered $H H$ system to (closed) channels containing vector 
heavy mesons makes the binding of two pseudoscalar mesons more strong. Thus, the search of a possible molecular-type state in any heavy-meson system but the one $(H H)$ considered in the present paper requires much more extensive analysis, making possible conclusions substantially less unambiguous.

The existence of a flavor-exotic $H H$ tetraquark depends crucially on the value $g$ of the $H^{*} H \pi$ coupling constant. The latter is related to the width of the $H^{*} \rightarrow H+\pi$ decay and, since corresponding partial width is measured, on the total width $\Gamma$ of the $H^{*}$-meson. Provided $\Gamma$ is taken equal to the experimental upper limit, the CTPX induced forces alone are strong enough to produce a bound state of $B B$ mesons, while the $D D$ system is very close to support a bound state. Such bound states will manifest themselves as stable scalar mesons with the mass $M \approx 2 m_{H}$ and the flavor quantum number equal to \pm 2 (note that existence of a $H H$ bound state implies that the $\bar{H} \bar{H}$ system is also bound). The same conclusion holds true for the flavor-hidden $\bar{h} H$ states which have the same binding energy. It is relevant to note that CTPX induced flavor exotic molecular-type states emerge at about the same values of $g$ which ensure boundedness of flavor-hidden $\bar{H}^{*} H^{*}$ and $\bar{H}^{*} H$ states generated by one-pion exchange [4, 57,3]. At smaller values of $g$ molecular-type states with any heavy meson content are not expected to emerge. More complicated systems of heavy mesons where both one- and (correlated) two-pion exchanges are operative will be considered elsewhere. 


\section{REFERENCES}

[1] J.-P.Ader, J.-M.Richard and P.Taxil, Phys.Rev. D25 (1982) 2370

[2] H.J.Lipkin, Phys.Lett. B172 (1986) 242

[3] A.V.Manohar and M.B.Wise, Nucl.Phys. B399 (1993) 17

[4] N.A.Törnqvist, Phys.Rev.Lett. 67 (1991) 556

[5] N.A.Törnqvist, Zs.Phys. C61 (1994) 525

[6] M.Shmatikov, Nucl.Phys. A (in press)

[7] T.Ericson and W.Weise, Pions and nuclei, Oxford University Press, 1988

[8] G.E.Brown, In: Mesons in nuclei, vol.I, Eds. M.Rho and D.Wilkinson, North-Holland Publishing Company, Amsterdam 1976, p.329

[9] G.E.Brown and A.D. Jackson, The Nucleon-Nucleon Interaction, North-Holland Publishing Company, Amsterdam 1976, p.137

[10] H.-C.Kim, J.W.Durso and K.Holinde, Phys.Rev. C49 (1994) 2355

[11] D.Lohse, J.W.Durso, K.Holinde and J.Speth, Nucl.Phys. A516 (1990) 513

[12] B.Moussallam, Nucl.Phys. A429 (1984) 429

[13] M.B.Wise, Phys.Rev. D45 (1992) R2188

[14] ACCMOR collaboration, Phys.Lett. B278 (1992) 480

[15] A.A.Bolokhov, A.N.Manashov, V.V.Vereshagin and M.V.Polyakov, Phys.Rev. D50 (1994) 4713

[16] P.Colangelo, G.Nardulli, A.Deandrea, N. Di Bartolomeo, R.Gatto and F.Feruglio, Phys.Lett. B339 (1994) 151 